\title{
Laparoendoscopic Single-Site Surgery for Vesicorectal Fistula Repair
}

\author{
Piotr Jarzemski, MD, PhD, Marcin Markuszewski, MD, PhD, Sławomir Listopadzki, MD, PhD, \\ Marcin Jarzemski, MD, Marek Roslan, MD, PhD \\ Department of Urology, Jan Biziel Medical University Hospital, Bydgoszcz, Poland (Drs Jarzemski P, Listopadzki, \\ and Jarzemski $M$ ). \\ Department of Urology, Medical University of Gdańsk, Gdańsk, Poland (Drs Markuszewski and Roslan).
}

\begin{abstract}
Background and Objectives: Minimally invasive techniques have been introduced to decrease the morbidity related to standard laparoscopic procedures. One such approach is transvesical laparoendoscopic single-site surgery (T-LESS). We describe our clinical experience of using this technique for vesicorectal fistula (VRF) repair.

Description: In October 2013, we performed the T-LESS repair of a vesicorectal fistula of $5 \mathrm{~mm}$ diameter in a 72 -year-old man, in whom conservative treatment with temporary colostomy and Foley catheter placement had failed. The procedure was performed transvesically (percutaneous intraluminal approach) with a single-port device via a $15 \mathrm{~mm}$ incision made $20 \mathrm{~mm}$ above the pubic symphysis. Standard $10 \mathrm{~mm}$ optic and straight laparoscopic instruments were used. The fistulous tract was dissected and closed in two layers with a running, absorbable, barbed suture. A cystostomy tube was left in place for 22 days, and a Foley catheter for 1 week.

Results: The operation lasted $155 \mathrm{~min}$. Blood loss was minimal. No complications were observed. The postoperative period was uneventful. During a 5-week follow-up, the patient reported no involuntary discharge of urine into the rectum. A voiding cystourethrogram revealed no presence of VRF, and laboratory examination results were all within the normal range. The colostomy was closed after 4 months, and a 12-month follow-up confirmed the integrity of both the urinary and digestive tracts.
\end{abstract}

Conclusion: Although substantial development of the instruments and skills is needed, the T-LESS VRF repair appears to be feasible and safe. Nevertheless, further experience and observations are necessary.

Key Words: Laparoscopic surgery, Prostate cancer, Vesicorectal fistula.

Citation Jarzemski P, Markuszewski M, Listopadzki S, Jarzemski M, Roslan M. Laparoendoscopic Single-site surgery for vesicorectal fistula repair. CRSLS e2016.00017. DOI: $10.4293 /$ CRSLS.2016.00017.

Copyright $(2) 2016$ by SLS, Society of Laparoendoscopic Surgeons. This is an open-access article distributed under the terms of the Creative Commons Attribution-Noncommercial-ShareAlike 3.0 Unported license, which permits unrestricted noncommercial use, distribution, and reproduction in any medium, provided the original author and source are credited.

Address correspondence to: Piotr Jarzemski, MD, Department of Urology, Jan Biziel Medical University Hospital, Ujejskiego 75 Street, 85-168 Bydgoszcz, Poland.

Telephone: +48 523655306, Fax: +48 523712630. E-mail: urolog@jarzemski.pl

\section{INTRODUCTION}

Minimally invasive approaches, including laparoendoscopic single-site surgery (LESS) and natural-orifice transluminal endoscopic surgery (NOTES), have been developed recently, to decrease the morbidity related to standard laparoscopic procedures..$^{1,2}$ Laparoscopy is associated with a certain morbidity involving complications after port placement. In LESS procedures, the number of ports required is reduced; therefore, single-port tech- niques may decrease morbidity after multiport laparoscopic access and provide favorable cosmetic results.

A variation on the LESS approach is transvesical laparoendoscopic single-site surgery (T-LESS). Since its introduction in 2009, various ablative or reconstructive T-LESS procedures have been performed successfully.3-8 We present an application of the T-LESS technique for vesicorectal fistula (VRF) repair, with the use of standard laparoscopic instruments and barbed sutures. 


\section{DESCRIPTION}

In October 2013, we performed T-LESS repair on a $5 \mathrm{~mm}$ diameter vesicorectal fistula in a 72 -year-old man who had undergone laparoscopic radical prostatectomy for intermediate-risk (PSA, $8.5 \mathrm{ng} / \mathrm{mL}$; Gleason score, 7), localized prostate cancer 6 months earlier.

Two weeks after surgery, the patient presented with fecaluria, pneumaturia, and urinary tract infection; a VRF was confirmed by cystoscopy and colonoscopy. A temporary colostomy was performed, and a Foley catheter was placed in the bladder for 4 weeks.

During the subsequent 5 months, the VRF persisted, and the patient qualified for reconstructive surgery. Because our team was experienced in minimally invasive transvesical single-port procedures, we decided to repair the fistula using the T-LESS technique. We obtained informed consent from the patient and the approval of the local ethics committee.

The procedure was performed with the patient under general anesthesia and placed in the lithotomy position. A $15 \mathrm{~mm}$ longitudinal skin incision was made $20-30 \mathrm{~mm}$ above the pubic symphysis in the midline. In addition, a short (10 mm length) incision was made in the rectus fascia. The bladder was filled with $300 \mathrm{~mL}$ sterile $0.9 \%$ saline, and the trocar of the TriPort+ access system (Olympus Winter and IBE, GmbH, Hamburg, Germany) was introduced bluntly into the bladder through the skin incision, under cystoscopic control (Figure 1A, 1B). The rings of the system were fixed and matched exactly to the abdominal wall (Figure 1C). The saline was then removed, and the bladder was filled with carbon dioxide up to a pressure of $14 \mathrm{~mm} \mathrm{Hg}$.

Through the $12 \mathrm{~mm}$ channel of the TriPort+, a $10 \mathrm{~mm}$ rigid $0^{\circ}$ videolaparoscope (Karl Storz, Tuttlingen, Germany) was introduced, and the bladder was examined carefully. A VRF with a diameter of $5 \mathrm{~mm}$ was identified in the bladder trigone. The ureteral orifices were completely visualized, so there was no need to catheterize the ureters.

Standard laparoscopic instruments of $5 \mathrm{~mm}$ diameter were passed through the $5 \mathrm{~mm}$ channels of the TriPort + . The fistulous opening was separated circumferentially with a hook electrode (Karl Storz), and the mucosal margin was dissected aside to expose the fistulous tract passing to the rectal wall (Figure 2). Dissection was performed very carefully, to avoid damaging the sphincter. Good visibility in the T-LESS technique made it possible to perform this maneuver safely.

The rectal wall defect was closed tightly with an absorbable 3-0 unidirectional barbed running suture (The V-Loc 90;
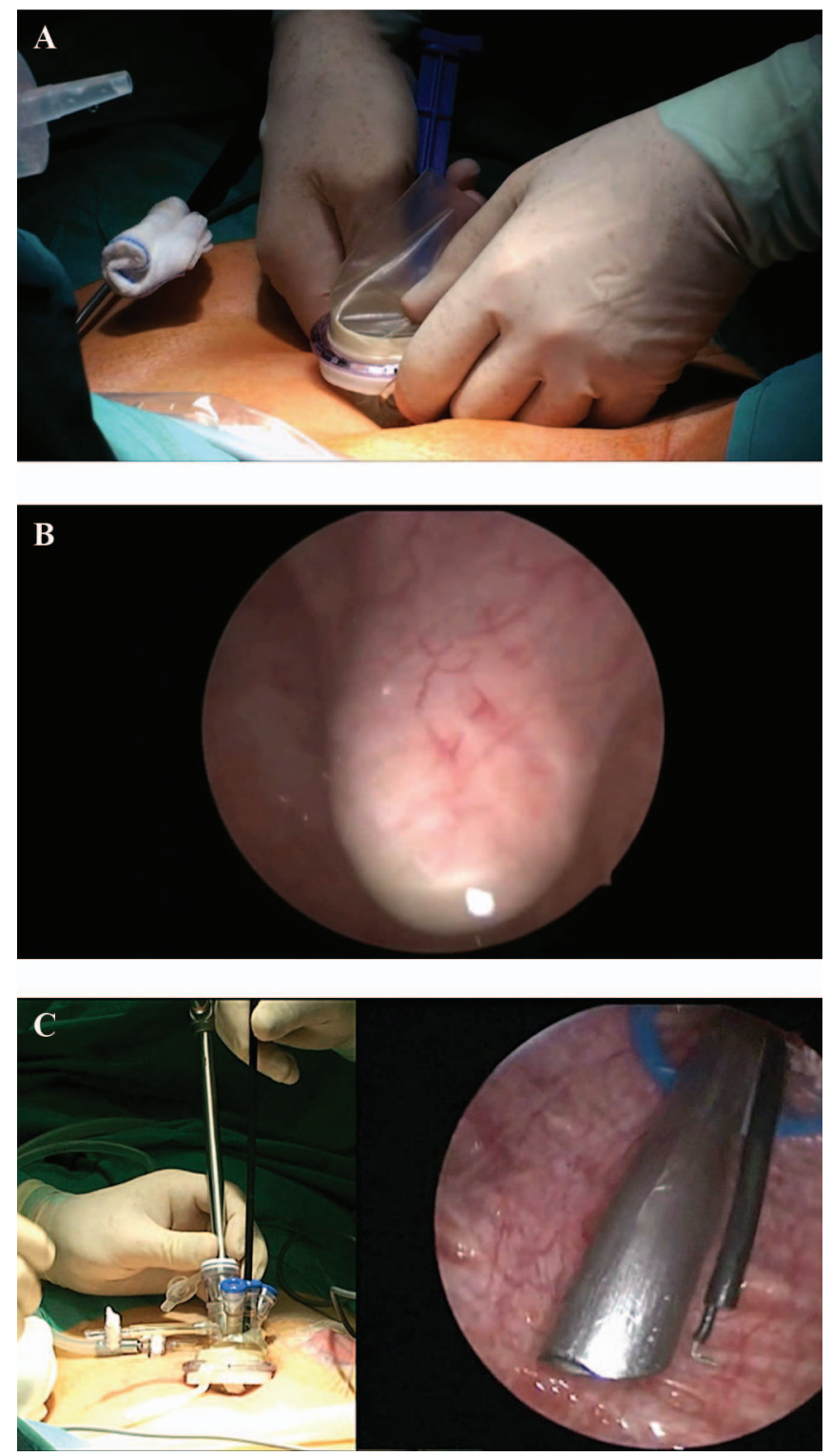

Figure 1. Establishing the TriPort+ for transvesical laparoendoscopic single-site surgery (T-LESS) for post prostatectomy vesicorectal fistula repair. (a) Insertion of an unbladed introducer. (b) Blunt passing of the introducer through the bladder wall. (c) Fixing the TriPort+ to the abdominal wall area.

Covidien, Norwalk, CT, USA; Figure 3). In this step, a laparoscopic grasper was introduced transurethrally to facilitate the suturing of the fistulous tract. Subsequently, the bladder wall defect was closed with another V-Loc suture.

At the end of the procedure, a cystostomy tube was left in place through the TriPort + channel and a Foley catheter was introduced transurethrally. The rectal sheath was closed with 


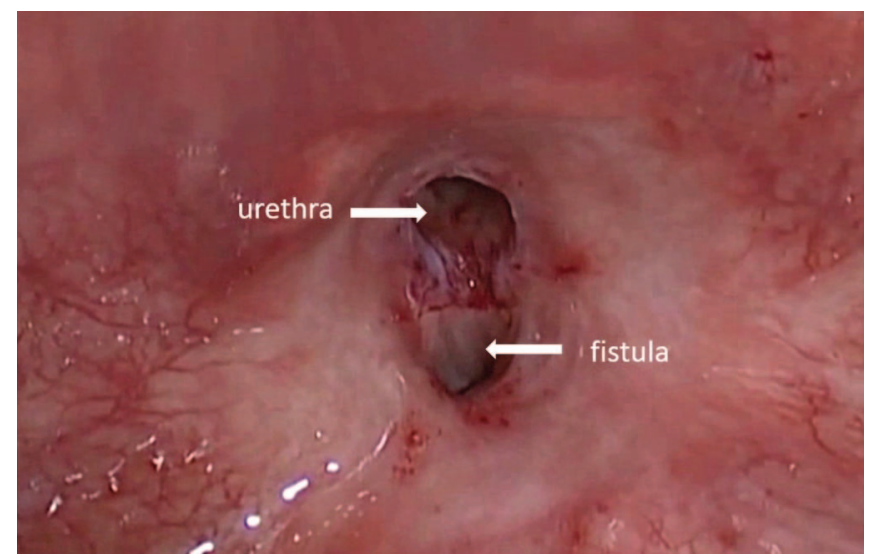

Figure 2. Dissection of the fistulous margin.

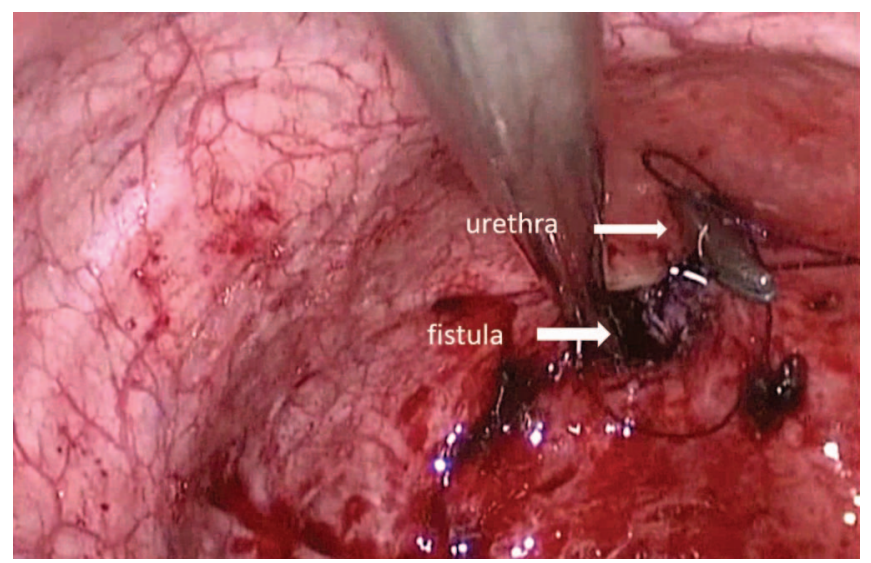

Figure 3. Closure of fistula edges using a running barbed V-Loc suture.

a single stitch and the skin incisions with 2 stitches. The opening in the bladder dome was not sutured. No other skin incisions or additional trocars were used.

The operating time was $155 \mathrm{~min}$. Blood loss was minimal. No intra- or postoperative complications were observed. The patient required 1 dose of ketoprofen $(50 \mathrm{mg}$, intramuscularly) on the first postoperative day. Perceived pain (measured on a 10-point visual analogue scale) decreased from 2 immediately after the operation to 1 on discharge. After the operation, the patient was administered intravenous cephalosporin. There was no reason for dietary restrictions, because a sigmoidostomy was performed before the operation. During the operation, preperitoneal access was used. On the first day after surgery, the patient received food. On postoperative day 3 , the patient was discharged home with a bladder catheter and cystostomy. The Foley catheter and the cystostomy tube were removed on postoperative days 7 and 22 , respectively.
Five weeks after discharge, a voiding cystourethrogram revealed no VRF. During a 4-month follow-up, the patient reported no involuntary leak of urine into the rectum. Laboratory examination results were all within normal ranges. The colostomy was subsequently closed and colon continuity was reestablished. At a 12-month follow-up, retrograde cystogram and computed tomography with rectal contrast reconfirmed the integrity of both the urinary and digestive tracts.

\section{DISCUSSION}

A VRF is one of the most devastating complications after radical prostatectomy. The incidence of VRF is $1-3.6 \%$ for radical retropubic prostatectomy (laparoscopic or open) and up to $11 \%$ for perineal prostatectomy. ${ }^{9}$ Although uncommon, VRFs cause substantial morbidity and significantly decrease the patient's quality of life.

Currently, there is no consensus on the best repair method for this condition; therefore, various open or minimally invasive techniques have been used to repair VRFs. The YorkMason approach is used most commonly to manage nonradiated fistulas. ${ }^{9}$ However, several advances in VRF repair techniques have been made over the past 10 years; these include laparoscopic, endoscopic, and robot-assisted procedures.

Sotelo et $\mathrm{al}^{10}$ initially described the application of the 5-port laparoscopic transperitoneal technique for VRF repair. In their case, the operative time was $240 \mathrm{~min}$, and the postoperative hospital stay was $3 \mathrm{~d}$. In 2008, the first description of robot-assisted laparoscopic rectovesical repair was also reported by Sotelo et $\mathrm{al}^{11}$ In that case, the operative time was $180 \mathrm{~min}$ and the postoperative hospital stay was $1 \mathrm{~d}$. In both patients, an interposition of the omentum on the rectal sutures was performed.

Successful management of a VRF with the endoscopic application of cyanoacrylate glue was reported by Bhandari et al in 2008. ${ }^{12}$ The use of small clips and tissue glue allowed Italian surgeons to perform a successful VRF repair. ${ }^{13}$ Nevertheless, these approaches using a sealant or glue are not yet commonly used for the treatment of VRFs or other fistulas.

Direct transvesical access for multiport laparoscopic VRF repair was described by Gözen et $\mathrm{al}^{14}$ in 2006. They inserted one $10 \mathrm{~mm}$ trocar for the optic and 2 additional $3 \mathrm{~mm}$ pediatric trocars into the bladder. They closed the fistula with a layer of running sutures, with no other tissue repositioning, and left in place either a Foley or a suprapubic catheter after surgery. The authors emphasize that for urologists familiar with endoscopic procedures, the 
presented approach provided excellent visibility for identification, excision, and repair of the fistula.

Several videos have been published recently demonstrating the application of percutaneous transvesical access for the repair of different urinary fistulas. ${ }^{15-18}$ The combination of robotic transperitoneal and transvesical accesses for VRF repair was presented by Modh et $\mathrm{al}^{15}$; this approach facilitated access to the intravesical opening of the fistulous tract. Moreover, according to Tobias-Machado et al, ${ }^{18}$ the transvesical approach provides additional potential benefitsnamely, less pain, and shorter recovery.

All the new transvesical techniques for VRF repair described above were performed with multiport procedures. A natural modification of these approaches is to use single-port access. As our team was experienced in T-LESS procedures for the treatment of pathologies including sling erosions, bladder diverticula, vesicoureteral reflux, and vesicovaginal fistulae, we decided to perform the VRF repair using T-LESS access. The procedure was successful and without complication, and the operative time of 155 min and the hospital stay of $3 \mathrm{~d}$ were comparable to results obtained using multiport transvesical access.

In summary, we consider the transvesical single-port approach for VRF repair to be feasible and provide perfect visualization inside the bladder. Moreover, because the operation is performed in a separate cavity that is devoid of structures in danger of inadvertent injury, as in the abdomen, the T-LESS can be even safer than single- or multiport techniques performed via the peritoneal cavity. Additional benefits are the potential for less postoperative pain and better cosmesis. We also believe this technique has all the advantages of other minimally invasive procedures and may be a stepping-stone for investigation of similar techniques.

The T-LESS technique for VRF repair proved to be a feasible and safe procedure that is worthy of consideration as an alternative approach for minimally invasive treatment of this condition. Nevertheless, further observations of a larger number of patients and the development of applicable instruments are needed.

\section{References:}

1. Kaouk J, Haber GP, Goel GR, et al. Single-port laparoscopic surgery in urology: initial experience. Urology. 2008;71:3-6.

2. Zhu J. Scarless endoscopic surgery: NOTES or TUES. Surg Endosc. 2007;21:1898-1899.

3. Ingber M, Stein R, Rackley R, et al. Single-port transvesical excision of foreign body in the bladder. Urology. 2009;74:13471350 .
4. Desai MM, Fareed K, Berger AK, et al. Single-port transvesical enucleation of the prostate: a clinical report of 34 cases. BJU Int. 2010;105:1296-1300.

5. Sotelo R, Rao P, Garza R, et al. Expanding indications of transvesical LESS surgery. Urology. 2011;78(suppl. 3A):159.

6. Roslan M, Markuszewski M, Kłącz J, Krajka K. Laparoendoscopic single-port transvesical diverticulectomy: preliminary clinical experience. J Endourol. 2012;26:975-979.

7. Roslan M, Markuszewski M, Kłącz J, et al. Laparoendoscopic single-site transvesical ureteroneocystostomy for vesicoureteral reflux in an adult: a one-year follow-up. Urology. 2012;80:719-723.

8. Bragayrac LN, Machuca V, Saenz E, et al. Transvesical laparoendoscopic single-site management of distal ureter during laparoscopic radical nephroureterectomy. J Endourol. In press.

9. Brandes SB. Complex urinary fistulas of the posterior urethra and bladder. In: Brandes SB, Morey AF, eds. Advanced Male Urethral and Genital Reconstructive Surgery. New York: Springer Science+Business Media; 2014;351-372.

10. Sotelo R, Garcia A, Yaime H, et al. Laparoscopic rectovesical fistula repair. J Endourol. 2005;19:603-607.

11. Sotelo R, de Andrade R, Carmona O, et al. Robotic repair of rectovesical fistula resulting from open radical prostatectomy. Urology. 2008;72:1344-1346.

12. Bhandari Y, Khandkar A, Chaudhary A, et al. Post-radical prostatectomy rectourethral fistula: endosopic management. Urol Int. 2008;81:474-476.

13. Mangiavillano B, Pisani A, Viaggi $P$, et al. Endoscopic sealing of a rectovesical fistula with a combination of an over the scope clip and cyano-acrylate injection. J Gastrointest Oncol. 2010;1:122-124.

14. Gözen AS, Teber D, Moazin M, Rassweiler J. Laparoscopic transvesical urethrorectal fistula repair: a new technique. Urology. 2006;67:833-836.

15. Modh R, Corbyons K, Su L-M, et al. Robotic repair of rectovesical fistula: combined anterior and posterior approach with omental flap interposition (video). J Urol. 2015;29. Available at http://online.liebertpub.com/toc/vid/29/3

16. Biewenga E, Santomauro M, Nork J, et al. Robot-assisted extraperitoneal transvesical repair of a colovesical fistula (video). J Endourol Part B Videourol. 2014;28. Available at http://online.liebertpub.com/toc/vid/28/6

17. Marotte JB, Alodia WM. Robot-assisted vesicovaginal fistula repair with excision of mesh and simultaneous intravesical ureteral reimplant (video). 31st World Congress of Endourology, New Orleans, LA, USA, 2013. Available at https:// www.youtube.com/watch?v=0epu5q_qIqw

18. Tobias-Machado M, Mattos PA, Juliano CA, et al. Transluminal approaches to vesicorectal fistula repair. Int Braz J Urol. 2014; 40:283-284. 